\title{
AVALIAÇÃO DA RECOMPOSIÇÃO DA COBERTURA VEGETAL DE DUNAS DE REJEITO DE MINERAÇÃO, EM MATARACA/PB
}

\author{
Douglas Antônio de Carvalho ${ }^{1}$ \\ Ary Teixeira de Oliveira-Filho ${ }^{2}$
}

Recebido em 28-10-92. Aceito em 30-09-93

\begin{abstract}
RESUMO - (Avaliação da recomposição da cobertura vegetal de dunas de rejeito de mineração, em Mataraca/PB). Realizou-se o levantamento fitossociológico da vegetação estabelecida sobre dunas de rejeito de mineração da Companhia Rutilo \& Ilmenita do Brasil (RIB) - na área de mineração do Guaju, no município de Mataraca, extremo norte do litoral do estado da Paraíba $\left(6^{\circ} 29^{\prime} \mathrm{S}, 34^{\circ} 56^{\prime} \mathrm{W}, 10\right.$ a $75 \mathrm{~m}$ de altitude), com o objetivo de subsidiar os trabalhos de recuperação ambiental, promovidos pela empresa. Foram registradas em 30 parcelas de $3 \times 3 \mathrm{~m}$, a composição florística e a estrutura (cobertura, sociabilidade e altura) da comunidade vegetal que se formou sobre a duna em dois setores diferentes: a) área onde houve a deposição de uma camada de $20 \mathrm{~cm}$ de solo de mata; b) área onde houve o plantio de quatro espécies pioneiras após adubação orgânica nas covas. No primeiro caso, encontram-se 51 espécies pertencentes a 21 famílias botânicas e no segundo 44 espécies pertencentes a 17 famílias. Os valores de cobertura, tanto para parcelas como para espécies foram maiores no tratamento que recebeu solo de mata.
\end{abstract}

Palavras-chave: Revegetação de dunas, fitossociologia.

ABSTRACT - (Evaluation of tailing sand dunes restauration in mining area, in municipality of Mataraca, Paraiba State). A phytosociological survey of the vegetation established on tailing sand dunes was carried out in the area of the mining company Rutilo \& Ilmenita do Brasil (RIB), municipality of Mataraca, northern coastland of the state of Paraiba, NE Brazil $\left(6^{\circ} 29^{\prime} \mathrm{S}, 34^{\circ} 56^{\prime} \mathrm{W}, 10\right.$ to $75 \mathrm{~m}$ of altitude). The purpose was to provide basic information to the environmental

1 - Departamento de Biologia, Escola Superior de Agricultura de Lavras, 37200-000, Lavras, MG.

2 - Departamento de Ciências Florestais, Escola Superior de Agricultura de Lavras 37200-000, Lavras, MG. 
restauration programs which have been implemented by the company. The floristic composition and the structure (percent cover, sociability and height) of the plant community that grew on the dune were registered in 30 plots with $3 \times 3 \mathrm{~m}$ in two different sectors: a) area where a pioneer plant community regenerated after the deposition of a $20 \mathrm{~cm}$ layer of forest topsoil; b) area where four pioneer species were planted after localized organic fertilization. In the first sector, 51 plant species of 21 families were found whilst in the other sector the figures were 44 species and 17 families. The cover values both for the plots and the species were higher in the sector covered by forest topsoil.

Key words: Environmental, restauration, coastal dunes, succession, weeds

\section{Introdução}

No extremo norte do litoral paraibano as retingas ocorrem sobre praias estreitas e dunas fixas. A companhia Rutilo \& Ilmenita do Brasil (RIB) realiza a mineração daquelas dunas e o material rejeitado pelo processo (dunas de rejeito) é depositado nas proximidades ou então no local onde primitivamente havia dunas fixas. A companhia, preocupada com a recuperação ambiental das áreas degradadas, vem testando alguns processos visando à estabilização das dunas de rejeito.

Relatos de experiências de revegetação de áreas degradadas por mineração, em regiões de condições adversas, como nas dunas costeiras, são encontrados para a Austrália (Brooks 1986; Clarke 1986; Scanlon \& Martinick 1986; Unwin \& Cook 1986) e Estados Unidos (Farmer et al. 1982; Maun \& Krajnyk 1989; Rapp et al. 1980). No Brasil, em termos de revegetação de dunas provenientes da atividade mineradora, este trabalho é pioneiro. A composição florística dos diferentes tipos fisionômicos de área de mineração da RIB foi objeto de trabalho anterior (Oliveira Filho \& Carvalho 1993).

Na presente comunicação são apresentados os resultados de um levantamento fitossociológico da vegetação secundária que se estabeleceu sobre uma das dunas de rejeito, com o propósito de avaliar dois processos de revegetação testados.

\section{Material e métodos}

Descrição da área

A Companhia Rutilo \& Ilmenita do Brasil (RIB) - Área de Mineração do Guaju, localiza-se no município de Mataraca, extremo norte do litoral do estado da Paraíba (6 $6^{\circ} 29^{\prime} \mathrm{S}, 34^{\circ} 56^{\prime} \mathrm{W}, 10$ a $75 \mathrm{~m}$ de altitude) (Figura 1). O clima é tropical e chuvoso, com uma curta estação seca (Tipo Am de Koeppen). A temperatura 
média anual é de $25,5 \mathrm{C}$ e as médias mensais oscilam de $23,7 \mathrm{C}$, em julho, a $26,8 \mathrm{C}$, em fevereiro. A precipitação média anual de $1796 \mathrm{~mm}$ concentra-se $85,7 \%$ de março a setembro; o mês mais seco ocorrendo em dezembro (média de $31 \mathrm{~mm}$ ) e os mais chuvosos em abril (média de $314 \mathrm{~mm}$ ) e julho (média de $315 \mathrm{~mm}$ ) (dados inéditos da RIB para o período de 1985-1991).

0 relevo da região é caracterizado por um sistema de dunas arenosas de origem marinha que se estende em pararelo ao mar, podendo atingir os $80 \mathrm{~m}$ de altitude em seu cume. Estas dunas são mineradas pela RIB, que extrai por via úmida em uma planta indústrial a pequena fração de grãos de ilmenita. Ao final do processo, formam-se grandes dunas de rejeito compostas de areia lavada, com níveis desprezíveis de matéria orgânica e sais minerais, sem banco de sementes e altamente sujeitas à erosão eólica.

Os trabalhos de revegetação destas dunas partiram de diversas tentativas feitas com o objetivo de se conhecerem as vantagens e desvantagens de um leque de opções técnicas. O presente trabalho foi conduzido na Duna de Rejeitos $\mathrm{n}^{\circ} 1$, onde foram desenvolvidos os trabalhos pioneiros de revegetação. Esta duna tem uma área de aproximadamente 5 ha $\mathrm{e}$ foi construída entre 1985 e 1987. Nesta duna foram testados dois processos de revegetação.

$\mathrm{Na}$ estação chuvosa de 1988 , uma vertente foi revegetada com plantio intercalado e no espaçamento de $0,30 \times 0,30 \mathrm{~m}$ de salsa roxa (Ipomoea pescaprae (L.) Sweet) - Convolvulaceae, fava de boi (Canavalia rosea (Sw.) DC.) - Fabaceae, grama-de-burro (Cynodon dactylon (L.) Pers.) e capim-gengibre (Paspalum maritimum Trin.) - Gramineae, com adubação orgânica na cova $(0,5 \mathrm{Kg}$ de bagaço de cana +esterco de gado + turfa, 1:1:1). Utilizaram-se propágulos vegetativos de mais ou menos $0,20 \mathrm{~m}$, coletados na região. Estas espécies foram escolhidas porque demosntram, coforme observações realizadas na região, uma grande habilidade para colonizar áreas arenosas perturbadas ou sob as condições de sucessão primária na restinga arenosa.

Na outra vertente foi esparramada, na estação chuvosa de 1989 , uma camada de $0,20 \mathrm{~m}$ de solo de mata (mata de restinga) existente na região. Este solo foi retirado das áreas a serem mineradas (logo após o seu desmatamento) usando-se trator de lâmina, amontoado e depois transportado à duna por caminhões-caçamba. ( $O$ processo de extração do minério é executado com a aprovação dos órgãos ambientais.) $\mathrm{O}$ espalhamento do solo sobre a duna foi realizado utilizando-se também trator de lâmina, sendo que nas vertentes mais inclinadas dois tratores funcionavam, ligados por cabode-aço.

Uma rede de irrigação, com aspersores espaçados de 12 a $18 \mathrm{~m}$, foi estabelecida sobre a duna. A freqüencia dos turnos de irrigação variou com as condições metereológicas, desde aspersão contínua na estação seca, a dois turnos diários na estação chuvosa. 


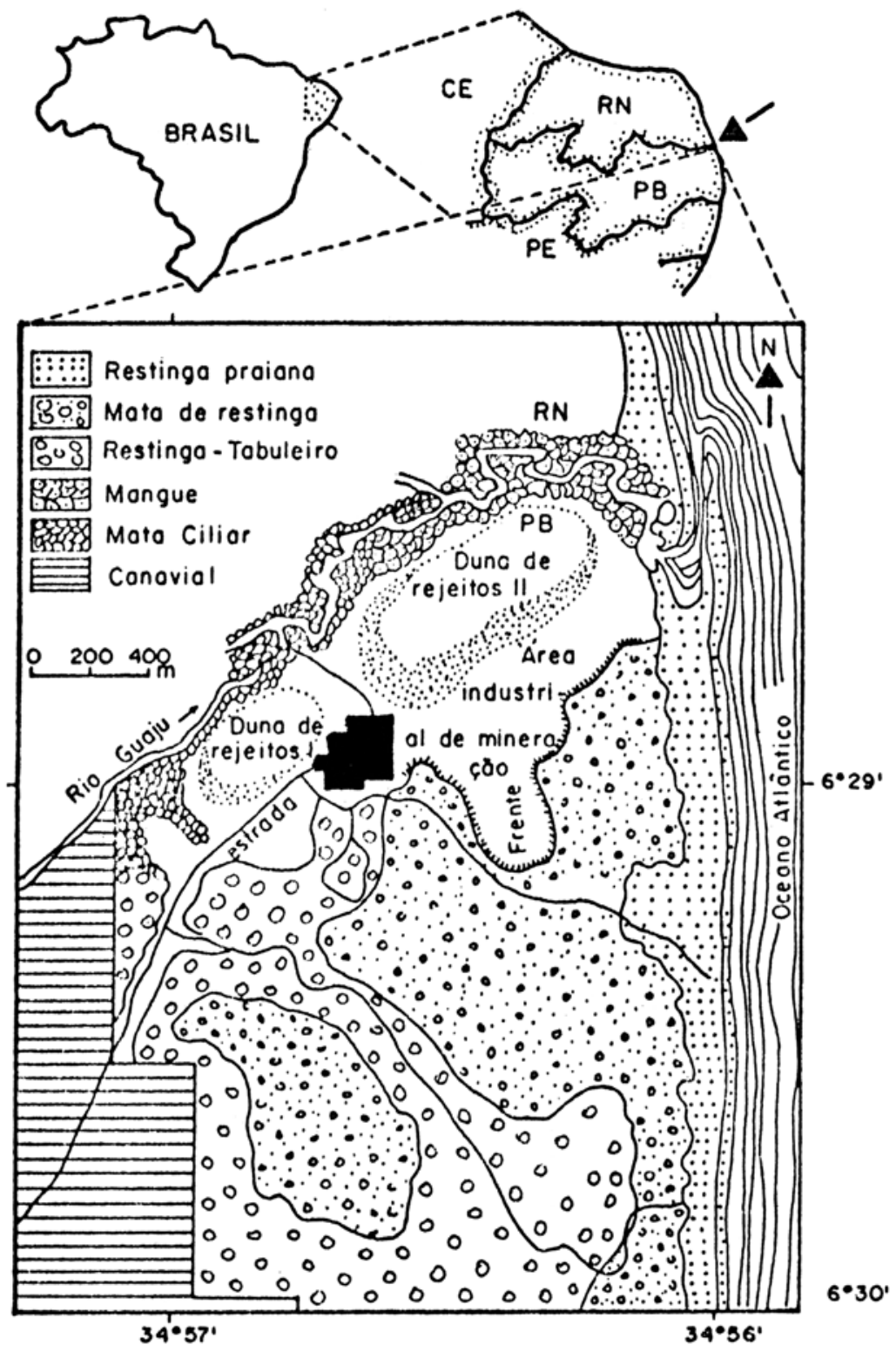

Figura 1 - Localização da área de mineração da Rutilo \& Ilmenita do Brasil (RIB) e da duna de rejeito $\left(\mathrm{n}^{\circ} 1\right)$. 
Os ventos alísios de sudeste predominam ao longo do ano. Ambos os tratamentos revestiam faces a barlavento e sotavento.

Levantamento florístico e estrutura

Tabela 1 - Escalas de magnitude do grau de cobertura e da sociabilidade, segundo Braun-Blanquet (1979)

\begin{tabular}{ccl}
\hline $\begin{array}{c}\text { Escala de } \\
\text { Magnitude }\end{array}$ & $\begin{array}{c}\text { Grau de } \\
\text { Cobertura (\%) }\end{array}$ & \multicolumn{1}{c}{ Sociabilidade } \\
\hline+ & $<1$ & \\
1 & $1 \mathrm{~A} 10$ & indivíduos isolados (caules ou troncos isolados) \\
2 & $10 \mathrm{~A} 25$ & em pequenos grupos (tufos) \\
3 & $25 \mathrm{~A} 50$ & em grupos maiores \\
4 & $50 \mathrm{~A} 75$ & em colônias ou tapetes extensos \\
5 & $75 \mathrm{~A} 100$ & população contínua \\
\hline
\end{tabular}

Realizaram-se, em junho de 1991, coletas de material botânico na duna de rejeito, já quase que totalmente coberta de vegetação. Em cada um dos tratamentos foram demarcadas, casualmente, 15 parcelas de $3 \times 3 \mathrm{~m}$, onde se avaliaram o grau de cobertura, a sociabilidade e a altura de cada planta (Tabela 1). Também 0 grau de cobertura de cada parcela foi avaliado.

O valor de cobertura (V.C.) para cada espécie foi calculado pela fórmula (Braun-Blanquet 1979):

V.C. = soma percentagens de cobertura da espécie nas parcelas $\times 100$

$\mathrm{n}^{\circ}$ total de parcelas

A freqüência das espécies foi determinada pela fórmula:

Freq. $=\mathrm{n}^{\circ}$ de parcelas em que a espécie ocorre $\mathrm{x} 100$

$\mathrm{n}^{\circ}$ total de parcelas

O material coletado foi identificado com o auxílio dos botânicos e das coleções dos herbários da Escola Superior de Agricultura de Lavras (ESAL), Reserva Ecológica do IBGE de Brasília (IBGE) e Universidade Estadual de Campinas (UEC). As exsicatas foram depositadas no herbário da Escola Superior de Agricultura de Lavras (ESAL).

\section{Resultados e discussão}

Nas tabelas 2 e 3 são apresentadas as espécies encontradas no presente levantamento, respectivamente nos tratamentos onde foi feito o plantio e onde foi 


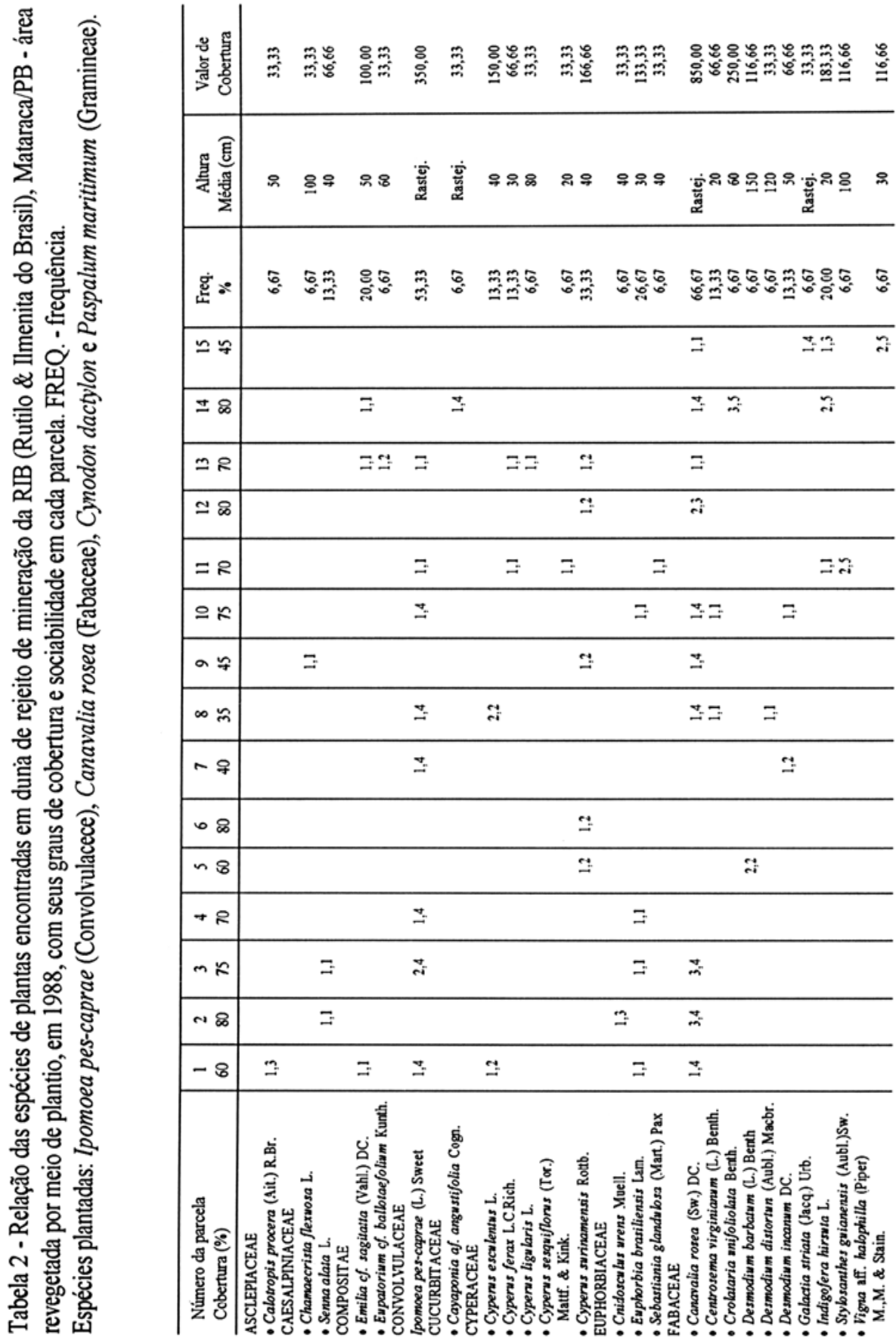




\begin{tabular}{|c|c|c|c|c|c|c|c|c|}
\hline 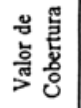 & 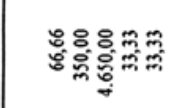 & : & $\stackrel{8}{0}$ & $\hat{2}=2$ & 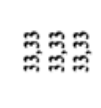 & : & 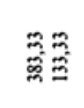 & 30 \\
\hline 兽 & 융유유응 & 㝘 & $\frac{\pi}{5}$ & 유요 & क्ष. & \& & 용 & $\therefore$ 요 \\
\hline 跑。 & 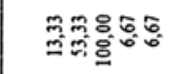 & 8 & $\stackrel{0}{0}$ & $\begin{array}{l}6.60 \\
60: 0\end{array}$ & $\begin{array}{l}6.65 \\
0.60\end{array}$ & $\stackrel{8}{\circ}$ & 85 & $5:$ \\
\hline$\simeq \approx$ & $\exists=\approx=$ & & & $=$ & & & $\cong$ & \\
\hline$\pm \infty$ & $\Xi=$ & $\Xi$ & & & & $=$ & $=$ & \\
\hline$m 2$ & $\approx=$ & \pm & $=$ & & \pm & $=$ & $a=$ & \\
\hline$\simeq \infty$ & $\Xi \approx$ & \pm & $\Xi=$ & & & $=$ & & \\
\hline$=R$ & $\approx=$ & \pm & & & : & & $=$ & \\
\hline 은 & $\approx$ & 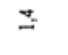 & & $=$ & & $=$ & $=$ & \\
\hline$a \cong$ & $\exists=$ & & & & & & & \\
\hline$\infty \approx$ & $\Xi=$ & \pm & & & & & & \\
\hline -9 & $\approx \approx$ & $=$ & & $\Xi$ & & & $=$ & \\
\hline$\circ \%$ & $=$ & 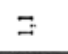 & & & & $=$ & $=$ & $=$ \\
\hline$n 8$ & $=$ & & & & & & $\Xi \approx$ & \\
\hline+2 & $=$ & & & & \pm & $=$ & & \\
\hline$m \approx$ & $=$ & & & & & & $\Xi$ & \\
\hline$\sim \infty$ & $\exists=$ & & & & & & $=$ & \\
\hline-8 & $\exists \triangle$ & $\Xi$ & $\Xi$ & & & & $=$ & $=$ \\
\hline 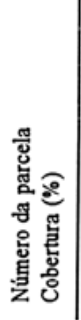 & 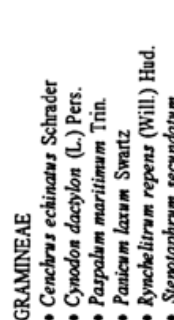 & 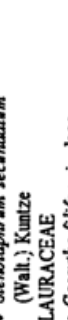 & 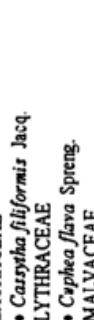 & 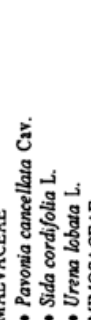 & 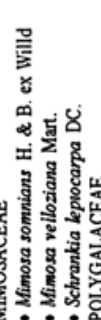 & 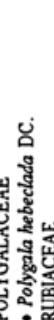 & 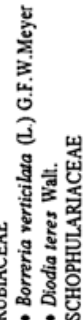 & 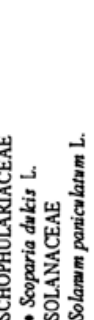 \\
\hline
\end{tabular}




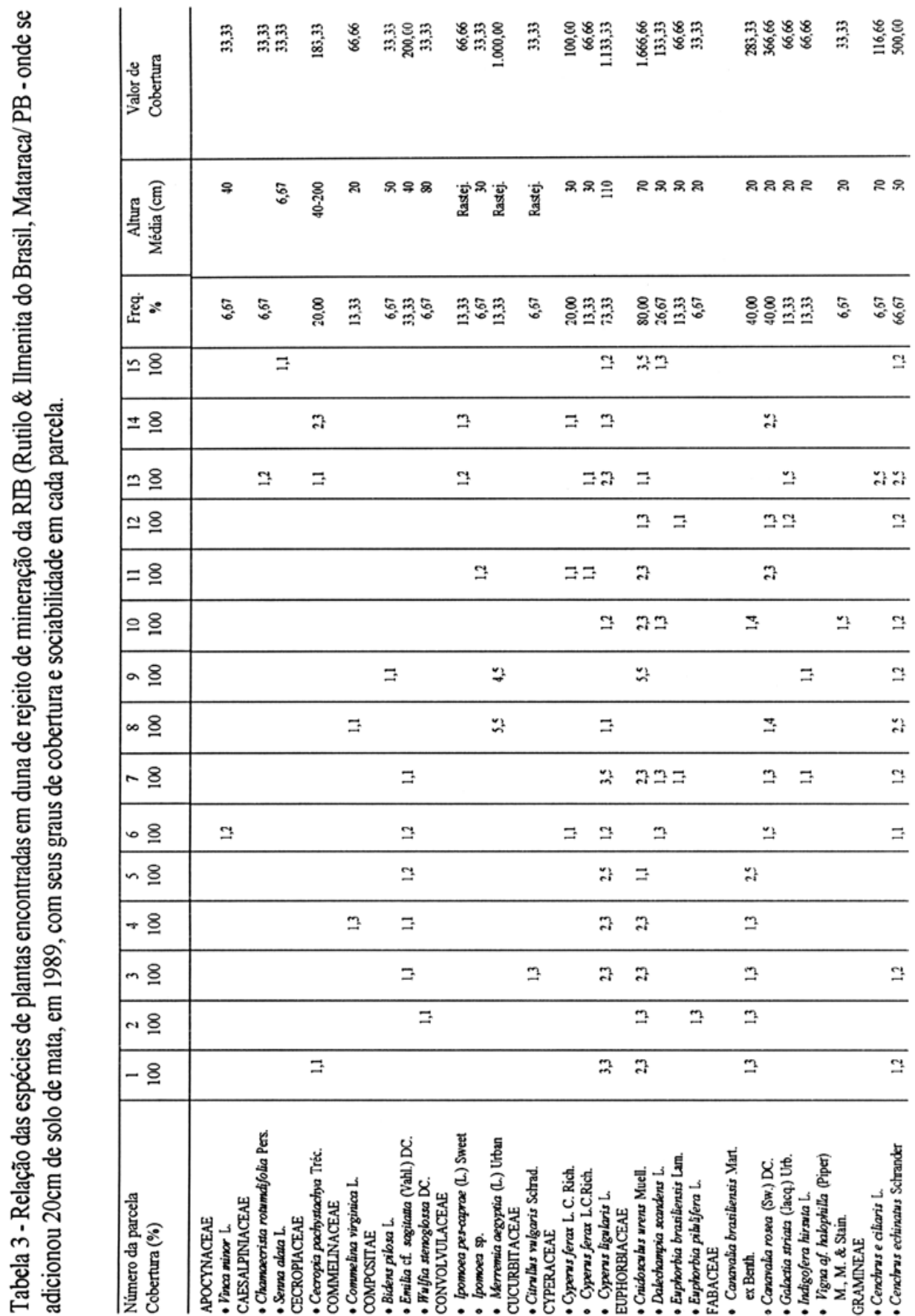




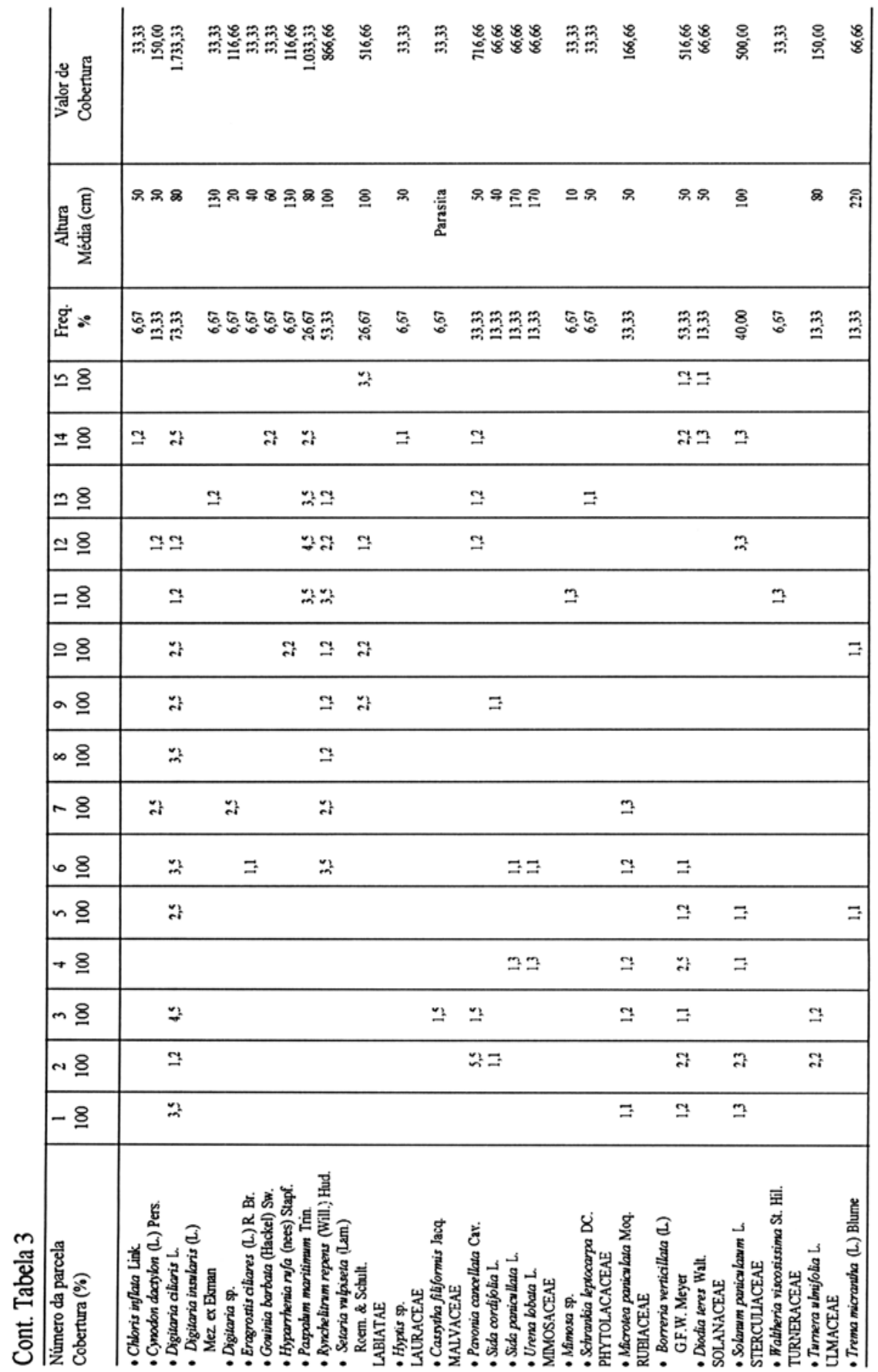


colocado o solo de mata. No primeiro caso foram encontradas 44 espécies pertencentes a 17 famílias botânicas; as famílias que apresentam maior número de espécies foram Euphorbiaceae, Mimosaceae e Malvaceae com 3 cada uma. As espécies mais freqüentes foram, em ordem decrescente, Paspalum maritimum, Canavalia rosea, Borreria verticillata, Stenotaphrrum secundatum, Ipomoea pescaprae, Cynodon dactylon e Polygala hebeclada; e as que apresentaram maior valor de cobertura, também em ordem decrescente foram Papalum maritimum, Canavalia rosea, Borreria verticillata, Cynodon dactylon, Ipomoea pes-caprae, Stenotaphrum secundatum, Crotalaria unifoliolata e Polygala hebeclada. Neste tratamento as espécies Canavalia rosea, Cynodon dactylon, Ipomoea pes-caprae e Paspalum maritimum foram plantadas, sendo que todas as demais surgiram espontaneamente.

Nas parcelas onde foi colocado solo de mata encontram-se 51 espécies pertencentes a 21 famílias botânicas; as famílias mais numerosas em espécies foram Gramineae, com 13, Fabaceae, com 5, Euphorbiaceae e Malvaceae, com 4 e Compositae e Convolvulaceae, com 3. As espécies mais freqüentes foram, em ordem decrescente, Cnidosculus urens, Cyperus ligularis, Digitaria ciliares, Cenchrus echinatus, Borreria verticillata, Rhynchelitrum repens, Canavalia brasiliensis, Canavalia rosea, Solanum paniculatum. As que apresentaram maior valor de cobertura, também em ordem decrescente, foram Digitaria ciliares, Cnidosculus urens, Cyperus ligularis, Paspalum maritimum, Merremia aegyptica, Rynchelitrum repens, Pavonia cancellata, Borreria verticillata, Setaria vulpiseta, Chenchrus echinatus e Solanun paniculatum. Todas as espécies deste tratamento surgiram espontaneamente na área.

No tratamento onde se colocou a camada de solo retirada da mata encontrouse um número de espécies $16 \%$ maior que na área plantada, sendo que nas 2 áreas as famílias que apresentaram de forma comum o maior número de espécies foram Gramineae e Fabaceae. Na área plantada, Fabaceae predominou e na área com solo de mata predominou Gramineae, indicando que as Fabaceae estão mais adptadas a colonizar ambientes praticamente estéreis como é a areia. As gramíneas, apesar de serem muitas vezes disseminadas pelo vento (que é forte na região), ficaram em segundo lugar nestas condições, mas foram mais abundantes na área onde foi colocado solo de mata. Isto pode ser explicado pela maior quantidade de sementes de gramíneas no solo de mata ou por estas encontrarem, quando trazidas pelo vento, condições de estabelecimento mais favoráveis nos locais onde previamente foi esparrado uma camada de $0,20 \mathrm{~m}$ de solo de mata sobre a areia.

As espécies,Borreria verticillata (Rubiaceae) e Canavalia rosea (Fabaceae) são comuns, no grupo das mais freqüentes (acima de $40 \%$ de freqüencia), nos 2 tratamentos. Borreria verticillata e Papalum maritimum (Gramineae) são comuns, no grupo das mais abundantes também nos dois tratamentos. Este resultado mostra que das espécies plantadas na área, Canavalia rosea e Paspalum maritimum corresponderam à expectativa de colonização e que Borreria verticilata 
deve ser considerada, a partir de então, uma espécie promissora para atividades de revegetação de dunas.

Os valores de cobertura das espécies, exceto para Paspalum maritimum, que foi plantada, foram maiores no tratamento com a camada de solo de mata. Também neste tratamento as parcelas apresentaram-se totalmente cobertas de vegetação - cobertura $100 \%$ - enquanto no outro esses valores variam de 35 a $80 \%$.

As espécies no tratamento com o solo de mata ocorreram em foram de mancha, ou seja, em uma área havia predomínio de uma espécie, a qual já não ocorria em outra área, onde o predomínio era de outra espécie.

\section{Referências bibliográficas}

Braun-Blanquet, J. 1979. Fitossociologia - bases para el estudio de las comunidades vegetales. H. Blume Ediciones, Rosário, Madrid. 820p.

Brooks, D.R. 1986. Land use and rehabilitation technology in the mineral sands industry. In: The Ausimm Perth Branch, Australia: a worls source of ilmenite, rutile, monazite and zircon. Conference... Austrália, 1986. p. 191-200.

Clarke, J. 1986. Environmental regulation in the Western Australian mineral sands mining industry. In: The Ausimm Perth Branch, Australia: a world source of ilmenite, rutile, monazite and zircon. Conference...Australia, 1986. p. 171-177.

Farmer Jr., R.E. Cunninghan, M. \& Barnhill, M.A. 1982. First year development of plant communities originating from forest topsoils placed on Souther Appalachian minesoils. Journal of Applied Ecology, 19:283-294.

Mauun, M.A. \& Krajynk, I. 1989. Stabilization of Great Lake (USA, Canadá) sand dunes; effect of planting time, mulches and fertilizer on seedling estabilishment. Journal of Coastal Research, 5(4):791-800.

Oliveira Filho, A.T. \& Carvalho D.A. 1993. Florística e fisionomia da vegetação do extremo norte do litoral da Paraiba. Revista Brasileira de Botânica, 16(1): $115-130$

Rapp; J.K.; Carrel, J.E.; Kucera, C.L.; Weems, S.R. \& Reese, G.A. 1980. Plant biomass production in missouri surface coal mines. Bulletin of the Ecological Society of América, 61:124.

Scanlon, T.J. \& Martinick, W.G. 1986. Environmental planning for mining of cables sands minninup deposit, Western Australia. In: The Ausimm Peth Branch, Australia: a world source of ilmenite, rutile, monazite and zircon. Conference... Australia, 1986. p. 211-213.

Unwin, G.L. \& Cook, J.B. 1986. Rehabilitation and environmental matters affecting mineral sands mining in New South Wales, Austrália. In: The Ausimm Perth Branch, Australia: a world source of ilmenite, rutile, monazite and zircon. Conference...Australia, 1986. p. 179-190. 